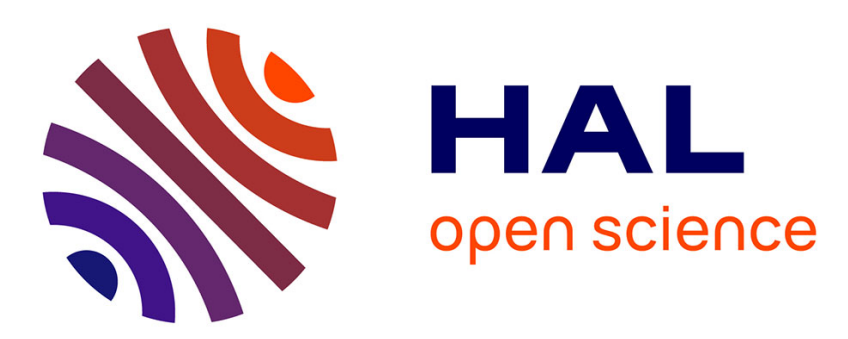

\title{
Analogies de méthodes et différences d'appareillage dans l'enregistrement d'informations corrélées
}

R. Ballini, E. Festa, J.P. Fouan, R. Kamoun, Franck Picard, B. Rosenbaum

\section{To cite this version:}

R. Ballini, E. Festa, J.P. Fouan, R. Kamoun, Franck Picard, et al.. Analogies de méthodes et différences d'appareillage dans l'enregistrement d'informations corrélées. Revue de Physique Appliquée, 1969, 4 (2), pp.119-120. 10.1051/rphysap:0196900402011900 . jpa-00243172

\section{HAL Id: jpa-00243172 https://hal.science/jpa-00243172}

Submitted on 1 Jan 1969

HAL is a multi-disciplinary open access archive for the deposit and dissemination of scientific research documents, whether they are published or not. The documents may come from teaching and research institutions in France or abroad, or from public or private research centers.
L'archive ouverte pluridisciplinaire HAL, est destinée au dépôt et à la diffusion de documents scientifiques de niveau recherche, publiés ou non, émanant des établissements d'enseignement et de recherche français ou étrangers, des laboratoires publics ou privés. 


\title{
ANALOGIES DE MÉTHODES ET DIFFÉRENGES D'APPAREILLAGE DANS L'ENREGISTREMENT D'INFORMATIONS GORRÉLÉES
}

\author{
R. BALLINI*, E. FESTA**, J. P. FOUAN*, R. KAMOUN*, \\ F. PIGARD** et B. ROSENBAUM**, \\ *Service de Physique Nucléaire à Basse Énergie, Saclay, \\ **Institut de Physique Nucléaire, Orsay.
}

\begin{abstract}
Résumé. - L'enregistrement des énergies de particules corrélées : expériences $(\alpha, \gamma)$, $\left({ }^{3} \mathrm{He}, \mathrm{d} \gamma\right),(\mathrm{d}, \mathrm{p} \gamma),\left(\mathrm{p}, \mathrm{p}^{\prime} \gamma\right)$, expériences de polarisation $[4,6,12]$, a été effectué indépendamment avec des appareillages de conceptions différentes. L'un est un système d'acquisition et de contrôle utilisant une logique câblée, l'autre un ordinateur en temps réel. L'examen critique de ces appareillages et de leur utilisation conduit à des conclusions voisines.
\end{abstract}

Abstract. - The acquisition of data from particle correlation experiments $(\alpha, \gamma),\left({ }^{3} \mathrm{He}, \mathrm{d} \gamma\right)$, $(\mathrm{d}, \mathrm{p} \gamma),\left(\mathrm{p}, \mathrm{p}^{\prime} \gamma\right)$, and polarization experiments $[4,6,12]$, has been carried out by means of different types of system, one based on hardware and others based on an on-line computer. Critical examination of each set-up leads to similar conclusions.

A titre d'exemple, une expérience de coïncidences entre un détecteur de particules chargées, avec identification par $\Delta E$, et l'un ou l'autre de deux détecteurs de $\gamma$, a pour but d'obtenir le spectre $\gamma$ d'états excités, sélectionnés par l'énergie et l'identité des particules chargées. La méthode la plus directe consiste à adresser à une mémoire incrémentale («bloc mémoire » par exemple) un mot numérique sélectionné par un pic du spectre d'identification et constitué par l'amplitude $\gamma$ et des «étiquettes»: deux bandes du spectre de temps $t_{\mathrm{p} \gamma}$, numéro du détecteur de $\gamma$, numéro d'un pic d'énergie.

Cette solution est insuffisante : un pic d'énergie peut ne pas être séparé des voisins; on peut même ignorer son existence et, par conséquent, ne même pas essayer de le sélectionner. C'est ce qui a conduit à faire tracer par un ordinateur (CAE 510) [1] les courbes de niveaux [2] de la surface lissée que définit le nombre $N\left(E_{\mathrm{p}} E_{\gamma}\right)$ de coïncidences, cote d'un point de coordonnées numériques $E_{\mathrm{p}}$ et $E_{\gamma}$. Mieux qu'une simple "visualisation XY », ce tracé permet au physicien d'utiliser, dans l'interprétation de ce qu'il voit, des critères de jugement très difficiles à programmer.

Le mot traduisant un événement est donc formé au moins de $E_{\mathrm{p}}, E_{\gamma}$ et d'étiquettes, soit une vingtaine de bits. L'incorporation d'autres paramètres dans ce mot peut être soit nécessaire (temps $t_{\mathrm{pr}}$ si l'on veut obtenir des vies moyennes), soit justifiée seulement par des raisons de sécurité (identification de particules) : il n'est pas exceptionnel qu'un mot de 40 à 50 bits soit ainsi formé. Dans ces deux derniers cas, l'usage d'une mémoire de masse telle qu'une bande magnétique est nécessaire pour un enregistrement chronologique (mot par mot) ou ordonné (par incrémentation).
I. Dispositifs utilisés à Orsay [3]. - Un ordinateur en ligne est à la base de deux structures techniques légèrement différentes, mais de logique identique. L'étude des niveaux de ${ }^{41} \mathrm{Ca}$ par corrélation $\mathrm{p}, \gamma$ [4] de la réaction ${ }^{40} \mathrm{Ca}\left(\mathrm{d}, \mathrm{p}^{\prime}, \gamma{ }^{41} \mathrm{Ca}\right.$ a été faite essentiellement sur notre premier système avec une IBM 1620 [5] et l'étude de la polarisation linéaire des $\gamma$ de la réaction ${ }^{12} \mathrm{G}\left({ }^{3} \mathrm{He}, \mathrm{p}\right){ }^{14} \mathrm{~N}[6]$ avec un PDP 8, utilisant le même ensemble et les mêmes programmes que pour l'expérience de corrélation $p, \gamma$. Dans les deux cas, la logique d'entrée et le filtrage des événements à conserver sont réduits à la discrimination en temps et en énergie. Le mot enregistré est formé de $E_{\mathrm{p}}, E_{\gamma}$ et d'étiquettes. Les appareillages sont décrits dans [3] et leurs caractéristiques sont les suivantes :

- 1620 IBM (40 000 décades). - Enregistrements chronologique et ordonné sur bandes magnétiques IBM. Cycle de mémoire de $20 \mu$ s, durée de paralysie de $20 \mathrm{~ms}$ par événement. Arrêt de l'acquisition par les opérations de contrôle (sortie par machine à écrire et traceur). Pas de visualisation oscilloscopique.

- PDP 8 (4096 mots de 12 bits). - Pas d'enregistrement chronologique. L'étendue de la mémoire ne permet que le stockage de spectres $\gamma$ corrélés avec plusieurs pics du spectre des particules. Cycle de mémoire de $1,5 \mu \mathrm{s}$. L'acquisition interrompt (300 $\mu \mathrm{s}$ environ) le programme principal de contrôle commandé par boutons-poussoirs : visualisation oscilloscopique, impression et perforation de spectres, traceur digital ( 1024 canaux en $30 \mathrm{~s}$ à la précision de $0,1 \mathrm{~mm}$ ) .

II. Dispositif utilisé à Saclay [7, 8]. - Ce dispositif a été utilisé dans diverses expériences sur l'émission $\gamma$ 
d'états excités [12]. Le mot traduisant un événement est regroupé dans des registres d'où il est transféré sur une bande magnétique de 16 pistes dont une de pilotage [7], soit en deux syllabes numérotées de 14 bits, soit en trois ou quatre de 13 bits. Le déroulement de la bande est normalement continu, de $6 \mathrm{~mm} / \mathrm{s}$ à $3 \mathrm{~m} / \mathrm{s}$. Le temps de paralysie, qui équivaut à $1 / 8 \mathrm{de} \mathrm{mm}$ de déroulement, impose l'usage d'une mémoire tampon $[9,10]$. La lecture ou le contrôle en temps réel reconstitue le mot initial si 0 ou 1 a été effectivement détecté sur chacun des 16 bits de chaque syllabe : les lectures défectueuses sont comptées, leur proportion excède rarement 2 à $3 \times 10^{-5}$, ce qui est acceptable pour un enregistrement mot par mot.

Les opérations de filtrage et de création d'étiquettes sont faites à différents niveaux, y compris à la sortie des registres de regroupement et après la lecture; à ce stade, le mot numérique est adressé à un conditionneur [7] qui examine simultanément si un nombre quelconque de paramètres répartis sur 52 bits vérifient les limites inférieure et supérieure imposées à chacun d'eux : les limites et la distribution des bits sont inscrites manuellement. Le mot ainsi réduit est transmis par une matrice à pions enfichables.

L'ordinateur CAE 510 [1] effectue aussi, en temps différé, l'opération de « conditionnement » à partir d'enregistrements spécialement préparés à partir de la bande initiale, pour des cas définis : par exemple, extraction de 18 spectres $\gamma$ de 1024 canaux corrélés avec 18 pics d'énergie des particules.

III. Examen critique de ces dispositifs. - Les points faibles du système avec 1620 sont surtout sa lenteur, l'interruption de l'acquisition par le contrôle, l'absence de visualisation oscilloscopique et l'utilisation de décimal codé binaire. Le principal point faible du système avec PDP 8 est l'absence d'une mémoire de masse (bande magnétique). Dans l'analyseur multiparamétrique de Saclay, l'exploitation différée des résultats exige un très grand nombre d'opérations manuelles, les montages réalisés pour le contrôle en temps réel ont tendance à être complexes, les bandes magnétiques ne sont utilisables que par un ordinateur pourvu d'un interface approprié, pratiquement le même que si l'information était prise avant l'écriture sur bande. Conçues pour l'usage en temps réel, des installations importantes comme celles de Yale et d'Orsay (voir compte rendu de ce Colloque) se prêteraient bien à l'exploitation d'enregistrements mot par mot (1) issus d'installations plus modestes et plus nombreuses, éventuellement extérieures au monde de la physique nucléaire et corpusculaire. L'enregistrement mot par mot, utilisé même dans le système à 1620 IBM où il n'était qu'une option, présente les principaux avantages suivants : possibilité de contrôler l'expérience (stabilité, élimination d'accidents) avant exploitation définitive, possibilité d'utiliser l'ensemble des résultats acquis pour effectuer certaines sélections (seuils) et certains calculs (identification de particules), exigences réduites sur la fiabilité de la lecture, permettant un matériel économique et d'entretien facile s'il était construit en assez grande série. L'enregistrement ordonné de mots de grand format par incrémentation sur une mémoire de masse [11], telle qu'un disque, serait plutôt à réaliser par le gros ordinateur en vue des nombreuses lectures que nécessite l'exploitation : s'il est effectué en temps réel (par exemple dans un but de contrôle) concurremment à l'enregistrement chronologique, l'enregistrement ordonné ne sera généralement pas utilisé tel quel après la fin de l'expérience.

Le contrôle en temps réel peut consister en une extraction partielle des résultats recherchés, mais on préfère généralement faire apparaître les effets les plus visibles témoignant du bon déroulement de l'expérience. Un ordinateur petit ou moyen (optimum vers $16 \mathrm{k}$ de $16 \mathrm{bits}$ ), rapide, pourvu de quelques fonctions câblées et d'une visualisation, mais pas nécessairement de moyens coûteux de sortie, est bien adapté à cet usage, ainsi qu'à la gestion de l'acquisition et à la préparation de l'exploitation définitive.

( $\left.{ }^{1}\right)$ Le support de l'enregistrement chronologique mot par mot n'est pas discuté dans cette note.

\section{BIBLIOGRAPHIE}

[1] Branchi (G.) et CorGE (C.), Note C.E.A., 1966, 621, p. 60.

DARONIAN (D.) et VeYriḱ-FAuCon (A.), ce colloque.

[2] Kamoun (R.), Faucon (A.) et Gluzman (P.), Nucl. Instr. Methods, 1968, 58, 45.

[3] Festa (E.), Picard (F.) et Rosennaum (B.), Coll. Intern. Électron. Nucl., 1968, 141.

[4] Johnson (G.), Laurent (H.), Picard (F.) et SchaPIRA (J. P.), J. Physique, 1968, 29, C1-92.

[5] Brun (J. C.), Verroust (G.) et Victor (C.), Ind. Atom., 1966, 11.

[6] Blake (R. S.), Johnson (G.), LAURENT (H.), SchaPIRA (J. P.) et PICARD (F.), Nucl. Phys., 1968, A 117, 561
[7] PAGES (A.), Nucl. Electronics, II, I.A.E.A., 1962, 185. Avril (M.), Hameau (P.), Joly (D.), Labreiy (J.), MOREAU (R.), PAGES (A.) et VIDAL (M.), Note C.E.A., 1966, 621, p. 48.

[8] BaliINI (R.), Cindro (N.), Deilaunay (J.), FouAN (J. P.), LoRet (M.) et PASSERIEUX (J. P.), Nucl. Phys., 1967, A 97, 561.

[9] Boucherie (A.), Thèse C.N.A.M., Paris, 1964.

[10] FrançoIs (B.) et al., Rapport Département d'Électronique, Saclay.

[11] Farvre (J. C.) et Escudié (J. L.), ce colloque.

[12] Kamoun (R.) et al., C. R. Acad. Sci., 1968, 266, 1241. BALIINI (R.), Cindro (N.), Delauday (J.), Fouan (J. P.), Nathan (O.) et Passerieux (J. P.), Phys. Letters, 1968, 26 B, 4.

TELLEZ (A.) et al., Nucl. Phys., 1969, A 127, 438. BEUZIT (P.) et al., Nucl. Phys., 1969, A 128, 594. 